\title{
The Wolf-Rayet population in the galaxy CTS 1026
}

\author{
Götz Gräfener ${ }^{1}$, Lars Koesterke ${ }^{2}$, and Wolf-Rainer Hamann ${ }^{1}$ \\ 1 Lehrstuhl Astrophysik, Institut für Physik, Universität Potsdam, \\ Am Neuen Palais 10, D-14469 Potsdam, BRD \\ 2 Laboratory for High Energy Astrophysics, \\ NASA Goddard Space Flight Center, Greenbelt, MD 20771, USA
}

\begin{abstract}
The blue compact H II galaxy CTS 1026 shows very strong WR emission features $\left(W_{\lambda}(4680)=17 \AA\right.$ and $\left.W_{\lambda}(5800)=10 \AA\right)$. We present high s/N optical spectra of the nucleus of this object, augmented by IUE UV spectra. By analysis of the WR profile shapes, we determine the dominant spectral types and the number of WN and WC stars in the star forming region. The number of O-type stars is determined by comparing the observed UV to optical flux distribution to population synthesis models.
\end{abstract}

\section{Introduction}

CTS 1026 has been identified as a WR galaxy by Peña et al. (1991), who performed a detailed nebular analysis. They found an oxygen abundance of $12+\log (\mathrm{O} / \mathrm{H})=8.3$, which implies a metallicity of $Z \simeq 0.004$, an interstellar extinction of $E_{B-V}=0.22 \mathrm{mag}$, and a number of ionizing $\mathrm{HI}$ photons of $Q_{0}=$ $5.5 \times 10^{52} \mathrm{~s}^{-1}$ (corresponding to $N_{\mathrm{O}} \mathrm{V}=5500$ stars) based on the redshift distance of $d=25.4 \mathrm{Mpc}$ for $z=0.0055$.

\section{WR profile analysis}

For the determination of the number of different WR subtypes we perform a detailed fit of the WR profiles by superposing observed single star spectra (Figure 1). This allows to disentangle the contributions of different stellar emission lines. The absolute numbers are then deduced from empirically determined mean line luminosities for single WR stars (Schaerer \& Vacca 1998).
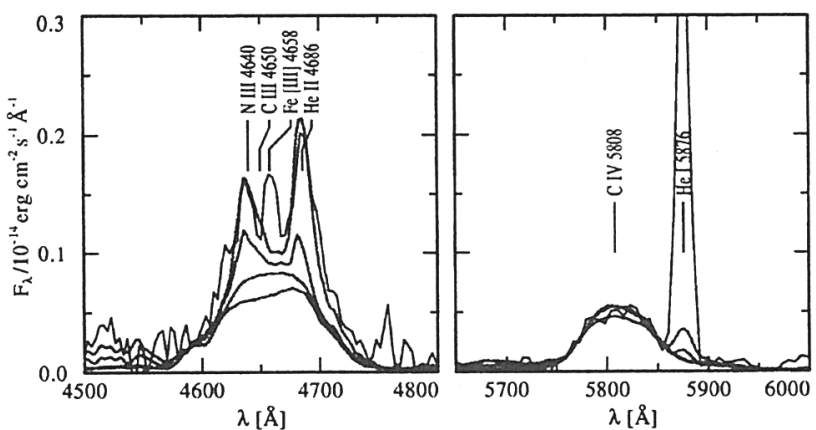

Figure 1. Fit of the observed WR profiles corrected for interstellar extinction (black) by accumulated fluxes of populations of spectral type WC 4, WC 5, WN 9, and WN 8 (grey). 


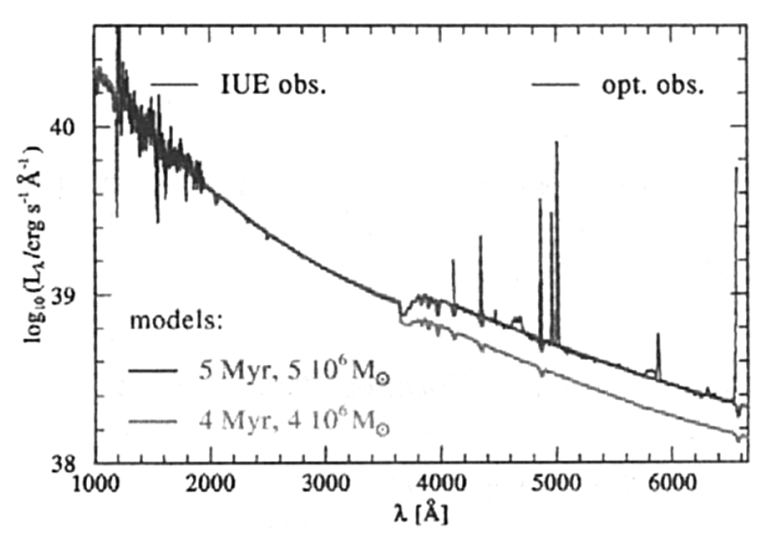

Figure 2. Comparison of the observed luminosity in the UV and optical corrected for interstellar extinction and distance (black) to STARBURST 99 population synthesis models for an instantaneous burst with $Z=0.004, \quad \alpha=2.35$ and $M_{\text {up }}=100 \mathrm{M}_{\odot}$ (grey).

We find that the WC population in CTS 1026 is dominated by $\sim 1000$ WCE stars close to subtype WC $4\left(L_{\mathrm{C} \text { IV } 5808}=3 \times 10^{39} \mathrm{erg} \mathrm{s}^{-1}\right)$. From the absence of CIII 5696 we determine an upper limit for the number of WC stars later than WC 5 of 85. To explain the high ratio of N III/He II $\left(L_{\mathrm{N} \mathrm{III}} 4640=1.3 \times 10^{39} \mathrm{erg} \mathrm{s}^{-1}\right.$, $L_{\mathrm{He} \text { II } 4686}=2.0 \times 10^{39} \mathrm{erg} \mathrm{s}^{-1}$ ) very late $\mathrm{WN}$ types are needed. Both values indicate a population of $\sim 1300$ WNL stars of spectral type WN8-9.

\section{Flux distribution}

The observed UV to optical flux distribution (Figure 2) is in good agreement with STARBURST99 population synthesis models (Leitherer et al. 1999) for an instantaneous burst at the corresponding metallicity of $Z=0.004$. The two models shown are both lying in the time span between 3 and $5 \mathrm{Myr}$ where a large number of WR stars is present. The models predict an ionizing flux of $\log \left(Q_{0}\right)=52.6-53.4$, in agreement with the observed value of 52.7.

The number of O-type stars in both models lies in the range of 14000 19000 , i.e., the observed WR/O ratio of 0.13 slightly exceeds the expected value of $\sim 0.1$. For the WC/WN ratio the models give values around 2.5 which abruptly drop to zero at $\sim 4.3 \mathrm{Myr}$. Therefore, even the observed ratio of $\mathrm{WC} / \mathrm{WN}=0.8$ can be explained by assuming a short but finite duration of the burst.

\section{Conclusions}

The strong WR emission features in CTS 1026 indicate a population of $\sim 1000$ WCE stars and $\sim 1300$ very late WN stars. The observed flux distribution and the derived number ratios are in agreement with instantaneous burst models of an age of $4-5 \mathrm{Myr}$, yielding a number of $\sim 17000$ O-type stars.

\section{References}

Leitherer, C., Schaerer, D., Goldader, J., et al. 1999, ApJS 123, 3

Peña, M., Ruiz, M.T., Maza, J. 1991, A\&A 251, 417

Schaerer, D., Vacca, W.D. 1998, ApJ 497, 618 